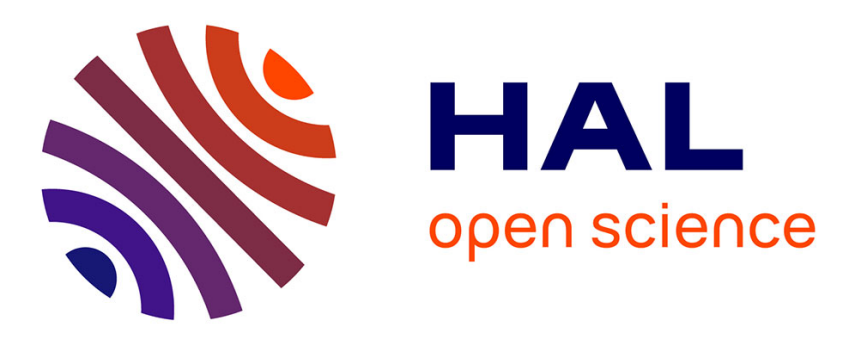

\title{
Building Reconfigurable Circuitry in a Biochemical World
}

\author{
Hui-Ju Chiang, Jie-Hong Jiang, François Fages
}

\section{To cite this version:}

Hui-Ju Chiang, Jie-Hong Jiang, François Fages. Building Reconfigurable Circuitry in a Biochemical World. IEEE Proceedings BioCAS 2014 - Biomedical Circuits and Systems Conference, Oct 2014, Lausanne, Switzerland. pp.560 - 563, 10.1109/BioCAS.2014.6981787 . hal-01103266

\section{HAL Id: hal-01103266 https://hal.inria.fr/hal-01103266}

Submitted on 14 Jan 2015

HAL is a multi-disciplinary open access archive for the deposit and dissemination of scientific research documents, whether they are published or not. The documents may come from teaching and research institutions in France or abroad, or from public or private research centers.
L'archive ouverte pluridisciplinaire HAL, est destinée au dépôt et à la diffusion de documents scientifiques de niveau recherche, publiés ou non, émanant des établissements d'enseignement et de recherche français ou étrangers, des laboratoires publics ou privés. 


\title{
Building Reconfigurable Circuitry in a Biochemical World
}

\author{
Hui-Ju Katherine Chiang*†, Jie-Hong R. Jiang*, and François Fages ${ }^{\dagger}$ \\ ${ }^{*}$ Graduate Institute of Electronics Engineering, National Taiwan University, Taipei, Taiwan \\ $\dagger$ Inria Paris-Rocquencourt, France \\ Email: \{d01943033@ntu.edu.tw; jhjiang@ntu.edu.tw; Francois.Fages@inria.fr\}
}

\begin{abstract}
Realizing complex systems within a biochemical environment is a common pursuit in synthetic biology, an emerging technology with promising potential in biomedicine and other applications. Such systems achieve certain computation through properly designed biochemical reactions. Despite fruitful progress being made, most existing reaction designs have fixed target functionality. Their lack of reconfigurability can be disadvantageous, especially when a system has to adapt to a varying biochemical environment. In this paper, we propose an analog approach to economically construct a reconfigurable logic circuit similar to a silicon based field programmable gate array (FPGA). The effective "logic" and "interconnect" of the circuit can be dynamically reconfigured by controlling the concentrations of certain knob species. We study a potential biomedical application of our reconfigurable circuitry to disease diagnosis and therapy at a molecular level.
\end{abstract}

\section{INTRODUCTION}

A synthetic approach to biology has been shown useful in biomedicine, energy, environment, and other applications. The advancements of synthetic biology have been broadening the range of realizable systems of increasing complexity both in vivo and in vitro. Building systems within a biochemical world is not far from reach and has been intensively studied, e.g., in terms of digital logic operations [6], [8], [14], analog computation [4], linear control [2], [10], signal processing [9], program flow control [7], etc. The bio-compatibility of such systems is unique in that they can embed computation tasks, including sensing, information processing, and actuation, inside living cells without physical intrusion. They are thus attractive in biomedical applications in disease diagnosis and therapy at a molecular level.

Most, if not all, of the engineered biochemical systems mentioned above have fixed specific functions or parametric values, and cannot be changed after design. This prespecification of functions or parameters can be disadvantageous when the underlying environment evolves over time with uncertainty or when the intended system behavior cannot be fully determined in the design phase. Even for electronic system design, which is very predictable, it is still not uncommon that a design has to be rectified even after it is manufactured. Likewise in biochemical system design, reconfigurability can be beneficial and crucial especially for biochemical environments, which are intrinsically stochastic. While the reconfigurability of integrated circuits (ICs) can be achieved through embedding firmware or programmable gate arrays into the design, it remains unclear how a similar mechanism can be economically deployed in a biochemical design.

In this paper, we propose a reconfigurable system, comprised of configurable logic units and interconnects, which are built from biochemical reactions. Our construction is advantageous in the following three respects. First, a configurable logic unit is made out of just a few reactions and species based on analog computation. Second, the function of a logic unit can be easily configured by altering the concentrations of certain biochemical species, similar to how organisms adapt their inner functions according to environmental signals received. Third, our construction maintains modularity and composability. The retroactivity [13] issue is overcome in the system, that is, composing a system with an extra module cannot invalidate the system's behavior. Finally, we discuss candidate implementation techniques and study the potential use of our reconfigurable circuitry in biomedical applications. Simulation results suggest the feasibility of our methods.

\section{PRELIMINARIES}

This paper adopts the classical chemical kinetic (CCK) model of biochemical reactions. It is assumed that molecules involved in reactions are of large quantities, so the spatial nonuniformity of molecule distribution becomes negligible, and the intrinsically stochastic reactions can be safely assumed to happen continuously and deterministically. Under the assumption, the attempt to use a set of ordinary differential equations (ODEs) to approximate the dynamic behavior of a biochemical system is justified.

Consider the following biochemical reaction

$$
\sum_{i=1}^{n} \alpha_{i} r_{i} \stackrel{k}{\rightarrow} \sum_{j=1}^{m} \beta_{j} p_{j},
$$

where species $r_{i}$ is the $i^{\text {th }}$ reactant and $p_{j}$ the $j^{\text {th }}$ product, coefficients $\alpha_{i}$ 's and $\beta_{j}$ 's specify the stoichiometric amounts, and $k$ is the rate constant. The species' dynamics under the CCK model is:

$$
k \prod_{i=1}^{n}\left[r_{i}\right]^{\alpha_{i}}=-\frac{1}{\alpha_{i}} \frac{d\left[r_{i}\right]}{d t}=\frac{1}{\beta_{j}} \frac{d\left[p_{j}\right]}{d t},
$$

where $\left[p_{j}\right]$ represents the concentration of species $p_{j}$.

In the sequel, to simplify notation, we do not distinguish a species and its concentration when they are clear from the context. Moreover, from a system perspective, we treat a species as a signal and the concentration of a species as the (non-negative) value of the corresponding signal.

\section{RECONFIGURABLE CIRCUITRY}

Our reconfigurable circuitry consists of two kind of components: configurable logic units (Sec. III-A) and configurable interconnects (Sec. III-B). Each logic unit (similar to those in silicon FPGAs) has $k$ input ports/species and one or multiple output ports/species. It can realize a certain set of logic functions up to $k$ inputs. (In our discussion we set $k=2$ and let the realizable functions be AND, OR, XOR, and NOT.) The logic 


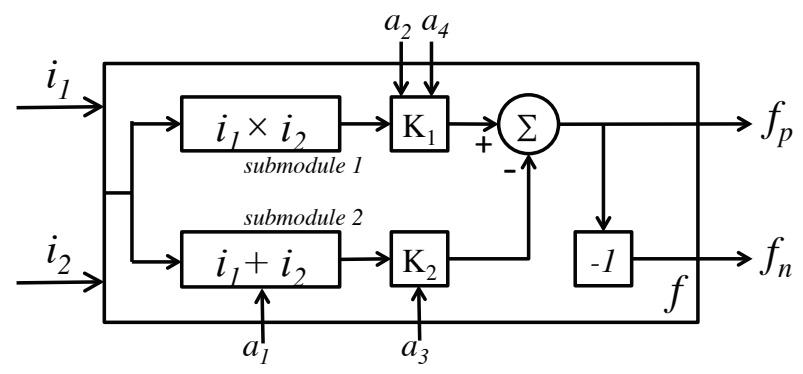

Fig. 1. Block diagram of configurable logic unit.

units can be composed through configurable interconnects. The construction of configurable logic units and interconnects is detailed as follows.

\section{A. Configurable Logic Units}

The configurable logic unit that we propose is realized through the following equations of arithmetic over reals.

$$
\left\{\begin{array}{l}
\operatorname{AND}\left(i_{1}, i_{2}\right)=-\left(0 \times\left(i_{1}+i_{2}\right)-1 \times\left(i_{1} \times i_{2}\right)\right) \\
\operatorname{OR}\left(i_{1}, i_{2}\right)=+\left(1 \times\left(i_{1}+i_{2}\right)-1 \times\left(i_{1} \times i_{2}\right)\right) \\
\operatorname{XOR}\left(i_{1}, i_{2}\right)=+\left(1 \times\left(i_{1}+i_{2}\right)-2 \times\left(i_{1} \times i_{2}\right)\right) \\
\operatorname{NOT}\left(i_{1}\right)=\operatorname{XOR}\left(i_{1}, 1\right)
\end{array}\right.
$$

The computation is depicted in the block diagram of Fig. 1. Two quantities, $\left(i_{1}+i_{2}\right)$ and $\left(i_{1} \times i_{2}\right)$, are common to the construction of all four considered logic functions, which differ only in the coefficients combining these two quantities and in the final sign. Assuming that the inputs $i_{1}$ and $i_{2}$ take on either 0 or 1 unit of concentration (signifying Boolean 0 or 1 logic value, respectively), one can verify that the four equations correspond to the four intended logic interpretations. In essence, the logic operations are achieved through arithmetic over reals, i.e., some form of analog computation, which can be more economical than the digital counterpart [4]. Notice that the definition of unit concentration is relative, and 0 and 1 units of concentration do not need to be exact; slight deviations in concentration from 0 and 1 are immaterial to the correctness of the interpretation.

Below we show how to implement the above four equations in terms of biochemical reactions. Essentially the four equations are implemented by the same set of reactions such that the output value of a configured logic unit coincides with the concentration at equilibrium of some designated species in the reactions. According to the block diagram of Fig. 1, the set of biochemical reactions is comprised of four groups:

(a) Reactions implementing Submodule 1 in Fig. 1:

$$
\left\{\begin{aligned}
i_{1}+i_{2} & \stackrel{k_{1}}{\longrightarrow} i_{1}+i_{2}+y_{1} \\
y_{1} & \stackrel{k_{2}}{\longrightarrow} \emptyset
\end{aligned}\right.
$$

(b) Reactions implementing Submodule 2 in Fig. 1:

$$
\left\{\begin{aligned}
a_{1}+i_{1} & \stackrel{k_{3}}{\longrightarrow} a_{1}+i_{1}+y_{2} \\
i_{2} & \stackrel{k_{4}}{\longrightarrow} i_{2}+y_{2} \\
y_{2} & \stackrel{k_{5}}{\longrightarrow} \emptyset
\end{aligned}\right.
$$

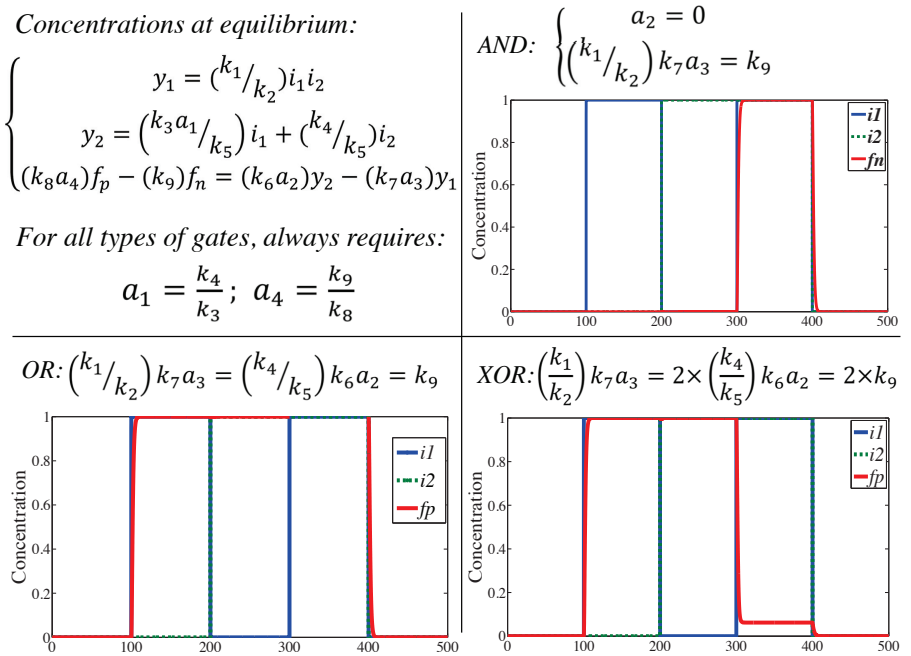

Fig. 2. Concentration settings and simulation results of the reconfigurable logic unit.

(c) Reactions implementing linear combination:

$$
\left\{\begin{array}{rlll}
a_{2}+y_{2} & \stackrel{k_{6}}{\longrightarrow} & a_{2}+y_{2}+f_{p} & (c .1) \\
a_{3}+y_{1} & \stackrel{k_{7}}{\longrightarrow} & a_{3}+y_{1}+f_{n} & (c .2) \\
a_{4}+f_{p} & \stackrel{k_{8}}{\longrightarrow} & a_{4} & (c .3) \\
f_{n} & \stackrel{k_{9}}{\longrightarrow} & \emptyset & (c .4)
\end{array}\right.
$$

(d) Reaction implementing output aggregation:

$$
f_{p}+f_{n} \stackrel{K}{\longrightarrow} \emptyset
$$

Given the above reactions, we are concerned with their equilibriums that we analyze as follows. Reactions (a.1) and (a.2) at equilibrium induce $y_{1}=\left(k_{1} / k_{2}\right)\left(i_{1} \times i_{2}\right)$ since

$$
\frac{d y_{1}}{d t}=k_{1} i_{1} i_{2}-k_{2} y_{1}=0 .
$$

Reactions (b.1), (b.2), and (b.3) at equilibrium induce $y_{2}=$ $\left(k_{3} a_{1} / k_{5}\right) i_{1}+\left(k_{4} / k_{5}\right) i_{2}$. Note that, in reaction (b.1), $a_{1}$ serves as an auxiliary species, whose purpose is to discharge the stringent rate matching to ensure $k_{3}=k_{4}$. With the presence of species $a_{1}$, reaction rates $k_{3}$ and $k_{4}$ obey $k_{3} a_{1}=k_{4}$, which can be easily satisfied since $a_{1}$ is a species with its concentration tunable externally. That is, we let $a_{1}=k_{4} / k_{3}$. Reactions (c.1), (c.2), (c.3), and (c.4) at equilibrium induce $\left(k_{8} a_{4}\right) f_{p}-\left(k_{9}\right) f_{n}=\left(k_{6} a_{2}\right) y_{2}-\left(k_{7} a_{3}\right) y_{1}$. Similarly, $a_{2}, a_{3}$, $a_{4}$ are auxiliary species whose concentrations can be controlled externally. Specifically, we let $a_{4}=k_{9} / k_{8}$, and let the concentrations of $a_{2}$ and $a_{3}$ be determined depending on the intended logic function (to be discussed). Effectively, species $a_{2}$ and $a_{3}$ serve as control knobs for function configuration. Finally, assuming $K$ much larger than other rate constants $k_{1}$, $\ldots, k_{9}$, reaction $(d .1)$ enforces one of output species $f_{p}$ and $f_{n}$ to have concentration 0 and the other to have concentration $\left|f_{p}-f_{n}\right|$.

By the above reactions, the function of a configurable logic unit can be altered by controlling the concentrations of species $a_{2}$ and $a_{3}$. Specifically, to configure an AND function, we set $a_{2}=0, a_{3}=\left(k_{2} k_{9}\right) /\left(k_{1} k_{7}\right)$ so that at equilibrium the output $f_{n}$ equals $\operatorname{AND}\left(i_{1}, i_{2}\right)$. To configure an OR function, we set 
$a_{2}=\left(k_{5} k_{9}\right) /\left(k_{4} k_{6}\right), a_{3}=\left(k_{2} k_{9}\right) /\left(k_{1} k_{7}\right)$ so that at equilibrium the output $f_{p}$ equals $\mathrm{OR}\left(i_{1}, i_{2}\right)$. To configure an XOR function, we set $a_{2}=\left(k_{5} k_{9}\right) /\left(k_{4} k_{6}\right), a_{3}=2\left(k_{2} k_{9}\right) /\left(k_{1} k_{7}\right)$ so that at equilibrium the output $f_{p}$ equals $\operatorname{XOR}\left(i_{1}, i_{2}\right)$. On the other hand, NOT function can be built from XOR. Therefore once inputs $i_{1}, i_{2}$ are assigned to their respective 0 or 1 values, the output converges to 0 or 1 automatically when the above reactions reach their equilibriums. Fig. 2 summarizes the concentration requirements and shows the simulation results under input sequence $\left(i_{1}, i_{2}\right)=(0,0),(0,1),(1,0),(1,1),(0,0)$ in a time separation of 100 units.

\section{B. Configurable Interconnects}

In addition to the four auxiliary input species, each configurable logic unit has two input $i_{1}, i_{2}$ and two output $f_{p}, f_{n}$ ports. These ports allow interconnection among multiple configurable logic units, and thus allow arbitrary composition of logic units to realize any Boolean function. We show how an interconnect can be made configurable as follows.

To have a configurable connection between a source port/species $s$ and a destination port/species $d$, we introduce a unique wiring species $w_{s d}$ for the pair such that $s$ and $d$ are connected (i.e., $[d]$ stabilizes to $[s]$ with negligible delay) if $w_{s d}$ is of value 1 (one unit concentration) and disconnected (i.e., $[d]$ resets to 0 regardless of $[s]$ ) if $w_{s d}$ is of value 0 (zero concentration). The reactions that fulfill this connection are:

$$
\left\{\begin{array}{rll}
s+a_{s d}+w_{s d} & \stackrel{k_{1}}{\longrightarrow} & d+s+a_{s d}+w_{s d} \\
d & \stackrel{k_{2}}{\longrightarrow} & \emptyset
\end{array}\right.
$$

where $a_{s d}$ is an auxiliary species making $a_{s d} \times k_{1}=k_{2}$ to discharge the need of rate matching of $k_{1}=k_{2}$, and the second reaction resets the destination species $d$ to 0 .

Notice that, unlike the well isolation of a signal in electronic circuits, a signal/species in a biochemical circuit without compartmental isolation is globally seen by all reactions. It is therefore necessary for each signal to be realized by a unique species. Note also that the retroactivity issue, similar to the loading effect in electronic circuits, is overcome in our construction by two means. First, the amount of an up-stream species is not affected by composing it with a down-stream species. For example, in reaction (e.1), up-stream species $s$ appears both as a reactant and a product with the same stoichiometric amount. Hence the amount of $s$ remains intact under the presence of reaction (e.1) for the creation of downstream species $d$. The same principle is applied to retain the amounts of species $i_{1}, i_{2}, a_{1}, a_{2}, a_{3}, a_{4}$ in the reactions $(a .1),(b .1),(c .1),(c .2)$. Second, we sustain the concentration of a species that can be consumed or produced by some reactions at its intended value based on equilibrium. For example, the concentrations of $f_{p}$ and $f_{n}$ remain at their equilibrium values due to the fact that the equilibriums of $y_{1}$ and $y_{2}$ are ensured by reaction groups $(a)$ and $(b)$ since no other reaction involves $y_{1}$ and $y_{2}$. Hence in the equilibrium equation $\left(k_{8} a_{4}\right) f_{p}-\left(k_{9}\right) f_{n}=\left(k_{6} a_{2}\right) y_{2}-\left(k_{7} a_{3}\right) y_{1}$, the righthand side is a constant and so are the values of $f_{p}$ and $f_{n}$ on the left hand side. (Species $a_{2}, a_{3}, a_{4}$ have determined constant concentrations.) Thereby our established modularity and composability ensure robust system construction.

\section{Logic Synthesis}

Given an arbitrary Boolean function, it can be realized with biochemical reactions by mapping it into our proposed reconfigurable architecture, similar to the conventional FPGA technology mapping in electronic design. Moreover, our approach well supports reconfigurable computing [3], [12] in biochemical systems. It is possible to conduct multiple computation tasks on the same circuitry in a time-multiplexed fashion and may have unique application in biochemical systems.

\section{CASE STUDY}

A microRNA (miRNA) is a small, highly conserved noncoding RNA that involves in almost every cellular process and down-regulates gene expressions through partial base-pairing with its (multiple) messenger RNA (mRNA) targets. Inappropriate miRNA expressions have been linked to the regulation and progression of a wide range of diseases [1], such as numerous cancers, cardiovascular, neurological, immunological, and metabolic diseases. Early onset of those diseases can be detected by monitoring changes in miRNA expression levels. Due to the partial base-pairing during target recognition, the regulation relation between miRNAs and mRNAs is many-tomany. As a result, diagnosis of certain diseases may involve multiple miRNAs and complex decision conditions, which may be expressible in Boolean formulae.

For potential implementation of our proposed biochemical reactions, there is recent demonstration of oligonucleotide AND-gates that can respond to specific miRNA inputs in live mammalian cells [6]. Moreover, DNA strand displacement [11], [15] has been successful in implementing various chemical reaction networks. These techniques may bring promise to the feasibility of conducting Boolean operations on miRNA inputs, recognizing endogeneuos miRNA expression patterns, and generating different oligonucleotide outputs correspondingly to manipulate miRNA levels for therapeutic purposes.

As reconfigurable circuitry may conduct different computation tasks utilizing the same set of reactions, it may realize different diagnostic and therapeutic strategies whichever one is needed. As a thought example, we consider function switching between two diagnostic-therapeutic specifications expressed in two Boolean expressions $f_{1}$ and $f_{2}$ :

$$
\begin{aligned}
& f_{1}=\left(x_{1} \vee x_{2}\right) \wedge\left(\neg x_{1} \vee x_{2} \vee \neg x_{3}\right) \wedge\left(x_{3} \vee x_{4}\right) \\
& f_{2}=\left(x_{1} \wedge x_{2} \wedge x_{3}\right) \vee\left(\neg x_{2}\right) \vee\left(\neg x_{1} \wedge x_{4}\right)
\end{aligned}
$$

where $\wedge, \vee, \neg$ are Boolean connectives conjunction (and), disjunction (or), and negation (not), respectively. Imagine that each variable $x_{i}$ represents a distinct type of miRNA related to the diagnostic tasks at hand. Let $f_{1}$ and $f_{2}$ encode the therapeutic actions corresponding to the diagnostic tests of diseases A and B, respectively. When disease A (resp. B) is in consideration, the reconfigurable circuitry implements $f_{1}$ (resp. $f_{2}$ ) function. The function output may be coupled with some miRNA whose expression level is to be raised for disease treatment.

A schematic diagram implementing the above two functions is shown in Fig. 3, where the gates correspond to the configurable logic units introduced in Sec. III-A, the four side-inputs to a gate indicate the auxiliary inputs, and the dashed boxes correspond to the configurable interconnects. For simplicity, here the configurability of interconnects is only limited to certain port to port connections. To implement functions $f_{1}$ and $f_{2}$ on the circuit shown, the inputs $\left(l_{1}, l_{2}, l_{3}, l_{4}, l_{5}, l_{6}, l_{7}, l_{8}, l_{9}\right)$ are assigned $\left(x_{1}, x_{2}, 0, \neg x_{1}, x_{2}, \neg x_{3}, x_{3}, x_{4}, 0\right)$ for $f_{1}$, and 


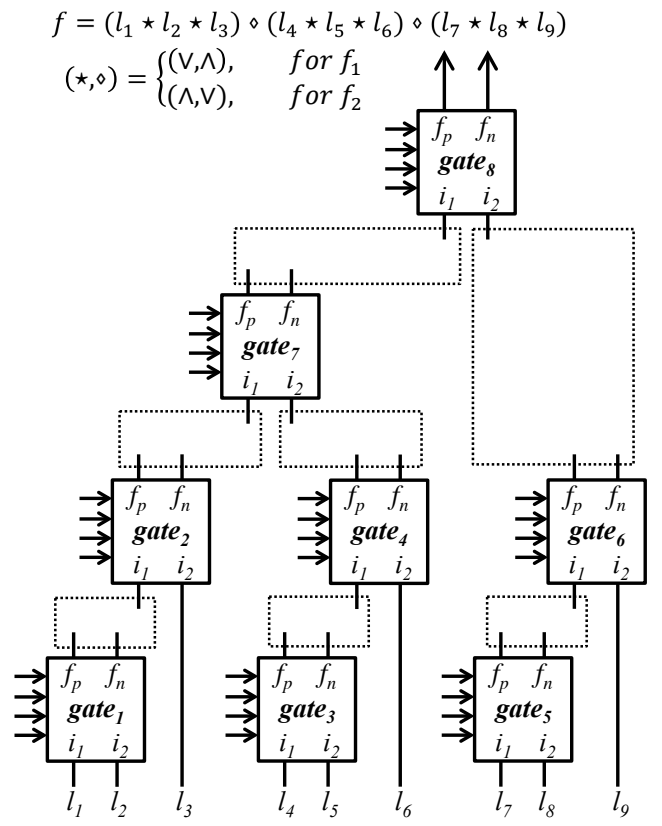

Fig. 3. A circuit diagram implementing functions $f_{1}$ and $f_{2}$.

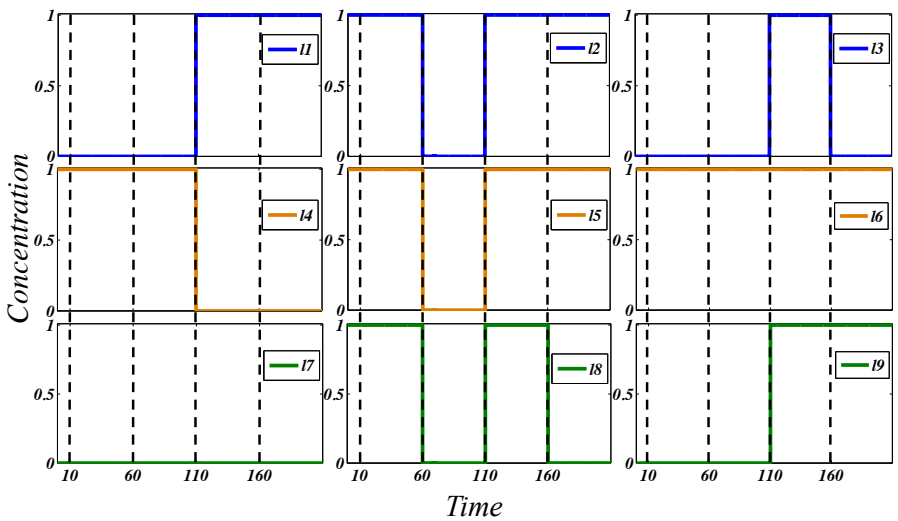

Fig. 4. Waveforms of inputs $l_{1}, l_{2}, \ldots, l_{9}$.

$\left(x_{1}, x_{2}, x_{3}, \neg x_{2}, 1,1, \neg x_{1}, x_{4}, 1\right)$ for $f_{2}$. Gates 1 to 6 implement the part of logic inside the parentheses in the formulae of $f_{1}$ and $f_{2}$, while gates 7 and 8 implement the logic operations that connect between parentheses. So for function $f_{1}$, the $f_{p}$ ports of gates 1 to 6 (which are of type OR) hold the evaluation results of formulae $\left(x_{1} \vee x_{2}\right),\left(x_{1} \vee x_{2}\right),\left(\neg x_{1} \vee x_{2}\right)$, $\left(\neg x_{1} \vee x_{2} \vee \neg x_{3}\right),\left(x_{3} \vee x_{4}\right)$, and $\left(x_{3} \vee x_{4}\right)$, respectively; the $f_{n}$ ports of gates 7 and 8 (which are of type AND) hold the evaluation results of $\left(x_{1} \vee x_{2}\right) \wedge\left(\neg x_{1} \vee x_{2} \vee \neg x_{3}\right)$ and $\left(x_{1} \vee x_{2}\right) \wedge\left(\neg x_{1} \vee x_{2} \vee \neg x_{3}\right) \wedge\left(x_{3} \vee x_{4}\right)$, i.e., function $f_{1}$, respectively. On the other hand, for function $f_{2}$, the $f_{n}$ ports of gates 1 to 6 (which are of type AND) hold the evaluation results of $\left(x_{1} \wedge x_{2}\right),\left(x_{1} \wedge x_{2} \wedge x_{3}\right),\left(\neg x_{2}\right),\left(\neg x_{2}\right)$, $\left(\neg x_{1} \wedge x_{4}\right)$, and $\left(\neg x_{1} \wedge x_{4}\right)$, respectively; the $f_{p}$ ports of gates 7 and 8 (which are of type OR) hold the evaluation results of $\left(x_{1} \wedge x_{2} \wedge x_{3}\right) \vee\left(\neg x_{2}\right)$ and $\left(x_{1} \wedge x_{2} \wedge x_{3}\right) \vee\left(\neg x_{2}\right) \vee\left(\neg x_{1} \wedge x_{4}\right)$, i.e., function $f_{2}$, respectively.

The above reconfigurable circuit is simulated using BIOCHAM [5]. The input waveforms and resultant output waveforms are shown in Fig. 4 and Fig. 5, respectively. The configuration switches from function $f_{1}$ to function $f_{2}$ at

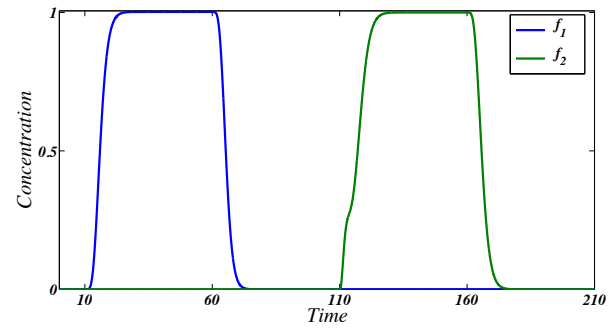

Fig. 5. Waveforms of outputs $f_{1}$ and $f_{2}$.

time $t=110$. After connection configuration is established at $t=10$, the input values change every 50 time units, at $t=60,110,160$, with input sequence $\left(x_{1}, x_{2}, x_{3}, x_{4}\right)=$ $(0,1,0,1),(0,0,0,0),(1,1,1,1),(1,1,0,0)$, which imitates the change of miRNA expression patterns. The waveforms of $l_{1}, \ldots, l_{9}$ in response to the input sequence is shown in Fig. 4. The waveforms of $f_{1}$ and $f_{2}$ are shown in Fig. 5, which imitate the therapeutic responses to diseases $\mathrm{A}$ and $\mathrm{B}$, respectively.

\section{CONCLUSION}

We presented a framework for building reconfigurable logic circuits using biochemical reactions. The reconfiguration can be easily done by controlling the concentrations of certain auxiliary species. In our construction, special attentions were paid to ensure modularity and composability. We studied potential biomedical application of our method in disease diagnosis and therapy at a molecular level. Our reconfigurable architecture may benefit system construction in synthetic biology for task switching in unpredictable environments.

\section{REFERENCES}

[1] J. A. Broderick and P. D. Zamore, "MicroRNA therapeutics," Gene Therapy, Vol. 18, No. 12, pp. 1104-1110, Apr. 2011.

[2] Y.-J. Chen et al., "Programmable chemical controllers made from DNA," Nature Nanotechnology, Vol. 8, pp. 755-762, Oct. 2013.

[3] K. Compton and S. Hauck, "An introduction to reconfigurable computing," IEEE Computer, Apr. 2000.

[4] R. Daniel et al., "Synthetic analog computation in living cells," Nature, Vol. 497, pp. 619-623, May 2013.

[5] François Fages et al., BIOCHAM 3.5 Reference Manual, Nov. 2013.

[6] J. Hemphill and A. Deiters, "DNA computation in mammalian cells: microRNA logic operations," J. Am. Chem. Soc, Vol. 135, No. 28, pp. 10512-10518, 2013.

[7] D.-A. Huang et al., "Compiling program control flows into biochemical reactions," in Proc. ICCAD, pp. 361-368, Nov. 2012.

[8] H. Jiang et al., "Digital logic with molecular reactions," in Proc. ICCAD, pp. 721-727, Nov. 2013.

[9] H. Jiang et al., "Digital signal processing with molecular reactions," IEEE Design \& Test of Computers, Vol. 29, No. 3, pp. 21-31, 2012.

[10] K. Oishi and E. Klavins, "Biomolecular implementation of linear I/O systems," IET Syst. Biol., Vol. 5, No. 4, pp. 252-260, 2011.

[11] D. Soloveichik et al., "DNA as a universal substrate for chemical kinetics," PNAS, Vol. 107, No. 12, pp. 5393-5398, 2010.

[12] T. J. Todman et al., "Reconfigurable computing: architectures and design methods," Computer and Digital Techniques, IEE Proceedings, Vol. 152, No. 2, pp. 193-207, Mar. 2005.

[13] D. Del Vecchio et al., "Modular cell biology: retroactivity and insulation," Molecular Systems Biology, Vol. 4, No. 161, 2008.

[14] L. Qian and E. Winfree, "Scaling up digital circuit computation with DNA strand displacement cascades," Science, Vol. 332, No. 6034, pp. 1196-1201, Jun. 2011.

[15] D. Y. Zhang and G. Seelig, "Dynamic DNA nanotechnology using strand-displacement reactions," Nature Chem., Vol. 3, No. 2, pp. 103$113,2011$. 